02

\title{
Фазовая диаграмма и особенности поведения мягких мод в твердых растворах со структурой хантита $\mathrm{TbFe}_{3-x} \mathrm{Ga}_{x}\left(\mathrm{BO}_{3}\right)_{4}$
}

\author{
(C) А.С. Крылов ${ }^{1}$, А.Н. Втюрин ${ }^{1,2}$, И.А. Гудим ${ }^{1}$, И.В. Немцев ${ }^{1,2,3}$, С.Н. Крылова ${ }^{1}$ \\ ${ }^{1}$ Институт фиизики им. Л.В. Киренского Сибирского отделения РАН - обособленное подразделение ФИЦ КНЦ СО РАН, \\ 660036 Красноярск, Россия \\ ${ }^{2}$ Сибирский фредеральный университет, \\ 660041 Красноярск, Россия \\ ${ }^{3}$ Федеральный исследовательский центр „Красноярский научный центр Сибирского отделения РАН“, \\ 660036 Красноярск, Россия \\ e-mail: shusy@iph.krasn.ru
}

Поступила в редакцию 22.07.2021 г.

В окончательной редакции 22.07.2021 г.

Принята к публикации 07.08.2021 г.

Выполнены исследования спектров комбинационного рассеяния четырех кристаллов твердых растворов $\mathrm{TbFe}_{3-x} \mathrm{Ga}_{x}\left(\mathrm{BO}_{3}\right)_{4}$ ( $x$ меняется от 0 до 0.54) в интервале температур от 8 до $350 \mathrm{~K}$. Установлены температуры структурных фазовых переходов в этих кристаллах. В низкочастотной области обнаружены процессы конденсации и восстановления мягких мод. Мягкие моды связаны со структурным фазовым переходом из фазы $R 32$ в фазу $P 3_{1} 21$ и испытывают изменения при магнитном переходе. Построена диаграмма зависимости температуры фазового перехода от содержания ионов галлия, частично замещающих ионы железа.

Ключевые слова: спектроскопия комбинационного рассеяния света, температурная зависимость, мягкие моды, ферробораты, фазовая диаграмма.

DOI: $10.21883 /$ OS.2022.01.51893.38-21

\section{Введение}

Кристаллы редкоземельных ферроборатов со структурой хантита [1] являются перспективными материалами для оптоэлектронных устройств. Например, в этом семействе обнаружены кристаллы с большими значениями магнитоэлектрического эффекта [2]. Такие кристаллы могут быть использованы в механизмах управления электрическими и магнитными свойствами материалов [3,4]. Сосуществование магнитных и сегнетоэлектрических параметров порядка приводит к тому, что кристаллы являются мультиферроиками $[5,6]$. Возможность практических применений и перспектива оптимизации характеристик путем частичного замещения редкоземельных ионов или ионов железа привлекает исследователей [7-12]. В частности, замена иона железа на ион галлия в кристалле $\mathrm{HoFe}_{3}\left(\mathrm{BO}_{3}\right)_{4}$ приводит к смещению температуры структурного фазового перехода [12].

Рост кристаллов со структурой хантитов, их первичная характеризация ранее были представлены в нескольких работах $[7,8,12]$. Эти исследования показали, что основными элементами структуры $R 32$ являются спиральные цепочки из октаэдров $\mathrm{FeO}_{6}$, направленные вдоль оси $c$. Впервые в работе [9] фазовый переход от структуры $R 32$ к $P 3_{1} 21$ в ферроборате тербия наблюдался при $198 \mathrm{~K}$. Следует отметить, что основным методом исследования в этой работе был метод ком- бинационного рассеяния света (КРС). В последующих исследованиях кристалла температура фазового перехода $192 \mathrm{~K}$ в $\mathrm{TbFe}_{3}\left(\mathrm{BO}_{3}\right)_{4}$ была определена другими методами [10,14,15].

Магнитный фазовый переход был обнаружен в кристалле $\mathrm{TbFe}_{3}\left(\mathrm{BO}_{3}\right)_{4}$ при температуре около $40 \mathrm{~K}$ [12] и исследовался различными методами [10,16-20]. Было установлено, что магнитные свойства кристалла определяются взаимодействием двух магнитных подсистем: элементов железа и тербия. При температуре $T_{\mathrm{N}}$ происходит антиферромагнитное упорядочение подсистемы железа с магнитными моментами, направленными вдоль оси третьего порядка $C_{3}$. В то же время антиферромагнитное упорядочение, вызванное взаимодействием с подсистемой железа, существует также в подсистеме тербия с магнитными моментами, параллельными той же оси. Кроме того, в ферроборате тербия индуцируется спин-переориентационный (магнитный) фазовый переход первого порядка $\left(H_{c}=35\right.$ Ое при $\left.4.2 \mathrm{~K}\right)$ [10,14,21]. Следует упомянуть работу Д. Саллера и др. [22], в которой авторы исследовали магнитные резонансы в кристалле $\mathrm{TbFe}_{3}\left(\mathrm{BO}_{3}\right)_{4}$. Они предположили, что существует шесть неэквивалентных магнитных подрешеток Fе. Для кристаллов $\mathrm{TbFe}_{3}\left(\mathrm{BO}_{3}\right)_{4}, \mathrm{NdFe}_{3}\left(\mathrm{BO}_{3}\right)_{4}, \mathrm{NdGa}_{3}\left(\mathrm{BO}_{3}\right)_{4}$, $\mathrm{HoGa}_{3}\left(\mathrm{BO}_{3}\right)_{4}$ также были выполнены теоретические расчеты, основанные на теории функционала плотности $[14,15,23,24]$. 
Таблица 1. Состав раствора-расплава (шихты) в квазибинарной форме и температура насыщения при синтезе твердых растворов $\mathrm{TbFe}_{3-x} \mathrm{Ga}_{x}\left(\mathrm{BO}_{3}\right)_{4}$

Состав раствора-расплава (шихты) в квазибинарной форме:

$(100-n) \operatorname{mass} \%\left[\mathrm{Bi}_{2} \mathrm{Mo}_{3} \mathrm{O}_{12}+p \mathrm{~B}_{2} \mathrm{O}_{3}+q \mathrm{~Tb}_{2} \mathrm{O}_{3}\right]+n \operatorname{mass} \% \mathrm{TbFe}_{3-x} \mathrm{Ga}_{x}\left(\mathrm{BO}_{3}\right)_{4}$

\begin{tabular}{c|c|c|c|c|c|c}
\hline \multicolumn{1}{c|}{ Кристалл } & $p$ & $q$ & $N$ & $T_{\text {sat }},{ }^{\circ} \mathrm{C}$ & $d T_{\text {sat }} / d n,{ }^{\circ} \mathrm{C} / \mathrm{mass} \%$ & $\Delta T_{\text {мет }},{ }^{\circ} \mathrm{C}$ \\
\hline $\mathrm{TbFe}_{3}\left(\mathrm{BO}_{3}\right)_{4}$ & 2.5 & 0.5 & 26 & 960 & 4.5 \\
\hline $\mathrm{TbFe}_{2.5} \mathrm{Ga}_{0.5}\left(\mathrm{BO}_{3}\right)_{4}$ & 2.5 & 0.5 & 27 & 948 & 4.0 \\
\hline $\mathrm{TbFe}_{2.45} \mathrm{Ga}_{0.55}\left(\mathrm{BO}_{3}\right)_{4}$ & 2.5 & 0.5 & 26 & 940 & 3.8 \\
\hline $\mathrm{TbFe}_{2.4} \mathrm{Ga}_{0.6}\left(\mathrm{BO}_{3}\right)_{4}$ & 2.5 & 0.5 & 26.7 & 960 & 3.8
\end{tabular}

Наиболее интересной при изучении структурных фазовых переходов является область низких колебательных частот, поскольку частота мягкой моды стремится к нулю при приближении к критической температуре, что вызывает неустойчивость кристаллической структуры. Впервые мягкая мода наблюдалась в одном из представителей семейства редкоземельных ферроборатов в кристалле $\mathrm{GdFe}_{3}\left(\mathrm{BO}_{3}\right)$ [25]. В дальнейшем методы колебательной спектроскопии света неоднократно использовались для исследования структурных и магнитных переходов в некоторых ферроборатах [13,25-32]. $\mathrm{B}$ кристалле $\mathrm{TbFe}_{3}\left(\mathrm{BO}_{3}\right)_{4}$ (без замещения ионов) были проведены температурные исследования тербия методом спектроскопии КРС $[9,18,33]$. При этом наблюдались аномалии в низкочастотной области колебательных спектров, связанные с магнитным фазовым переходом [9,33], а также аномалии в области средних частот, связанные со структурным фазовым переходом [18,33]. Стоит отметить, что мягкие моды в ферроборате тербия наблюдались не только в спектрах КРС, но и в спектрах инфракрасного поглощения в работе М.Н. Поповой с соавторами [34].

Можно ожидать, что при изменении температуры в твердых растворах $\mathrm{TbFe}_{3-x} \mathrm{Ga}_{x}\left(\mathrm{BO}_{3}\right)_{4}$ будут обнаружены аномалии в низкочастотной области спектров КРС. Целью настоящей работы являются исследования влияния замещения ионов железа на ионы галлия в твердых растворах $\mathrm{TbFe}_{3-x} \mathrm{Ga}_{x}\left(\mathrm{BO}_{3}\right)_{4}$ на температуру структурных фазовых переходов и поведение низкочастотных спектральных линий (мягких мод).

Авторы посвящают эту статью профессору Марине Николаевне Поповой по случаю ее 80-летия и за большой вклад, сделанный ею в исследованиях ферроборатов методами оптической спектроскопии [9,20,25,34,35].

\section{Эксперимент}

Монокристаллы $\mathrm{TbFe}_{3}\left(\mathrm{BO}_{3}\right)_{4}, \quad \mathrm{TbFe}_{2.5} \mathrm{Ga}_{0.5}\left(\mathrm{BO}_{3}\right)_{4}$, $\mathrm{TbFe}_{2.45} \mathrm{Ga}_{0.55}\left(\mathrm{BO}_{3}\right)_{4}$ и $\mathrm{TbFe}_{2.4} \mathrm{Ga}_{0.6}\left(\mathrm{BO}_{3}\right)_{4}$ (по закладке) выращены из раствора-расплава на основе $\left(\mathrm{Bi}_{2} \mathrm{Mo}_{3} \mathrm{O}_{12}-\mathrm{B}_{2} \mathrm{O}_{3}-\mathrm{Tb}_{2} \mathrm{O}_{3}\right)$ [36]. Соответствующие составы представлены в табл. 1 .
Раствор-расплавы массой $150 \mathrm{~g}$ готовили в платиновом цилиндрическом тигле $(D=40 \mathrm{~mm}, H=50 \mathrm{~mm})$ последовательным сплавлением при $1100^{\circ} \mathrm{C}$ оксидов $\mathrm{Bi}_{2} \mathrm{O}_{3}, \mathrm{MoO}_{3}, \mathrm{~B}_{2} \mathrm{O}_{3}, \mathrm{Fe}_{2} \mathrm{O}_{3}, \mathrm{Ga}_{2} \mathrm{O}_{3}, \mathrm{Nd}_{2} \mathrm{O}_{3}$.

На стадии растворения при этой же температуре перемешиваемый раствор-расплав выдерживали 8-10 h. Этого времени достаточно для его полной гомогенизации. Температура насыщения $T_{\mathrm{sat}}$ определялась с точностью $\pm 2{ }^{\circ} \mathrm{C}$ с помощью пробных кристаллов, которые предварительно получали на стержневом кристаллодержателе в режиме спонтанного зарождения. После завершающей гомогенизации температуру раствора-расплава снижали до $T_{\text {sat }}+7^{\circ} \mathrm{C}$, стержень с четырьмя затравками погружали в раствор-расплав и включали его реверсивное вращение со скоростью $30 \mathrm{rpm}$. Через $10 \mathrm{~min}$ температуру понижали до $T_{\mathrm{sat}}-10^{\circ} \mathrm{C}$ и далее плавно по программе с нарастающей скоростью $d T / d t=1-3^{\circ} \mathrm{C} /$ day. Рост продолжался от 5 до 14 days. За это время вырастали качественные кристаллы с размерами до 4-12 mm. Кристаллодержатель с выросшими кристаллами приподнимали над раствором-расплавом, и печь остывала до комнатной температуры с выключенным питанием. Первоначально были синтезированы кристаллы $\mathrm{TbFe}_{3}\left(\mathrm{BO}_{3}\right)_{4}, \mathrm{TbFe}_{2.5} \mathrm{Ga}_{0.5}\left(\mathrm{BO}_{3}\right)_{4}$, после изучения в них структурных фазовых переходов было принято решение о дополнительном синтезе кристаллов с составами $\mathrm{TbFe}_{2.45} \mathrm{Ga}_{0.55}\left(\mathrm{BO}_{3}\right)_{4}$ и $\mathrm{TbFe}_{2.4} \mathrm{Ga}_{0.6}\left(\mathrm{BO}_{3}\right)_{4}$. Разница во времени составляла несколько десятков месяцев.

Спектры КРС кристаллов были исследованы в диапазоне температур от 8 до $350 \mathrm{~K}$. Спектры были получены в геометрии обратного рассеяния с использованием спектрометра Horiba Jobin Yvon T64000 (Horiba, France) с тройным монохроматором, работающего в режиме вычитания дисперсии. Спектральное разрешение для записанных спектров стоксовой компоненты спектра КРС было $2 \mathrm{~cm}^{-1}$ (это разрешение было достигнуто при использовании решеток с $1800 \mathrm{lines} / \mathrm{mm}$ и щелями $100 \mu \mathrm{m}$ ). Разрешение низкочастотной области при исследовании мягкой моды было улучшено до $1.2 \mathrm{~cm}^{-1}$, что позволило достичь низкочастотного предела $10 \mathrm{~cm}^{-1}$. В качестве источника возбуждающего света использова- 
лись лазеры: одномодовый $\mathrm{Ar}+$ лазер $514.5 \mathrm{~nm}$ SpectraPhysics Stabilite 2017 (USA) мощностью $10 \mathrm{~mW}$ на образце $\left(\mathrm{TbFe}_{3}\left(\mathrm{BO}_{3}\right)_{4}, \mathrm{TbFe}_{2.5} \mathrm{Ga}_{0.5}\left(\mathrm{BO}_{3}\right)_{4}\right)$ и твердотельный одномодовый лазер Spectra-Physics Excelsior-532300-CDRH (USA) $532 \mathrm{~nm}$ и мощностью $5 \mathrm{~mW}$ на образце $\left(\mathrm{TbFe}_{2.45} \mathrm{Ga}_{0.55}\left(\mathrm{BO}_{3}\right)_{4}\right.$ и $\left.\mathrm{TbFe}_{2.4} \mathrm{Ga}_{0.6}\left(\mathrm{BO}_{3}\right)_{4}\right)$. Температурные эксперименты проводились на гелиевом криостате замкнутого цикла ARS CS204-X1.SS, управляемом термоконтроллером LakeShore 340. Контроль температуры осуществлялся калиброванным кремниевым диодом LakeShore DT-670SD1.4L. В качестве термоинтерфейса между холодным пальцем и образцом использовалась индиевая фольга. Измерения проводились в криостате, предварительно откачанном до давления $10^{-6} \mathrm{mbar}$. Во время температурного эксперимента возбуждающий луч совершал линейное сканирование по поверхности образца с максимальной амплитудой $0.3 \mathrm{~mm}$. Эта позволило, с одной стороны, усреднить эффекты, вызванные возможной неоднородностью нагрева, с другой стороны, снизить тепловую нагрузку благодаря более равномерному распределению нагрева образца лазером и, как в следствие, уменьшению погрешности измерения температуры. Эксперименты проводились в динамическом режиме, варьируя температуру образца. Скорость изменения температуры составляла $0.8 \mathrm{~K} / \mathrm{min}$. Неопределенность измеренной температуры для данной скорости можно оценить, как разницу между соседними измерениями. Общее время съемки одного спектра не превышало $30 \mathrm{~s}$. Спектры были измерены с шагом по температуре $0.4 \mathrm{~K}$. Исследуемые образцы представляли собой оптически прозрачные неориентированные монокристаллы зеленоватого цвета размером менее $3 \mathrm{~mm}$ с естественной огранкой и не содержали видимых под микроскопом дефектов и включений разной окраски. Температура структурного фазового перехода определялась по температурам аномалий в спектрах, включающих в себя появление новых линий в спектре КРС, и анализом поведения мягких мод. Температура перехода корректировалась с учетом нагрева образца лазерным излучением по методике, описанной в работе [31].

Для контроля элементного состава исследуемых кристаллов проводилась рентгеновская энергодисперсионная микроскопия (РЭМ) вышеуказанных кристаллов при ускоряющем напряжении $20 \mathrm{kV}$ на настольном сканирующем электронном микроскопе TM4000Plus (Hitachi, Япония), совмещенном с энергодисперсионным детектором XFlash Detector 630Hc (Bruker, Germany), позволяющем выполнять рентгеновское картирование (X-Ray Mapping). Перед микроскопией образцы не подвергались никакой дополнительной обработке: на их поверхность не наносилось тонких слоев металла либо углерода, чтобы не вносить дополнительных погрешностей в эксперимент. Пробоподготовка образцов заключалась в следующем. Производился свежий скол кристалла, затем с помощью двухсторонней углеродной токопроводящей ленты для РЭМ (Ted Pella, Inc., USA) образец монтировался на медную подложку для РЭМ, изготовленную из анодной меди, чтобы исключить возможные примеси при EDX-анализе. Ввиду наличия углеродной ленты для РЭМ завышено содержание углерода на рентгеновских картах. Поскольку микроскоп TM4000Plus работает в режиме детектора обратно рассеянных электронов (back scattered electrons, BSE), то карта представлена в формате фазового контраста, что дает возможность оценить однородность образца по химическому составу: чем элемент тяжелее (выше порядковый номер $Z$ ), тем он ярче. Анализ BSE-карт показал, что все исследованные кристаллы однородны, аномалий распределения элементов не обнаружено.

\section{Результаты и обсуждение}

При структурных фазовых переходах второго рода или переходах первого рода, близких к трикритической точке, частота одной или нескольких нормальных мод кристаллической решетки стремится к нулю или сильно уменьшается. Моду с самой низкой частотой с аномальным спадом обычно называют „мягкой модой““. Концепция и теория мягкой моды, а также терминология, связанная с конденсацией мягких мод и их последующим восстановлением в искаженной фазе после фазового перехода, рассматривалась в большом количестве теоретических и экспериментальных исследований. В короткой статье, посвященной спектральным исследованиям фазовых переходов в кристаллах хантитов, неуместно излагать историю этой области. Мы можем лишь сослаться на некоторые работы, как на хорошую отправную точку для изучения этого явления [37,38]. Отличный обзор спектроскопии мягких мод на ранней стадии подобных исследований дал Скотт [39].

При исследовании температурных спектров КРС в ряду твердых растворов $\mathrm{TbFe}_{3-x} \mathrm{Ga}_{x}\left(\mathrm{BO}_{3}\right)_{4}$ наше внимание привлекло необычное поведение мягких мод. Следует отметить, что в кристаллах $\mathrm{TbFe}_{3}\left(\mathrm{BO}_{3}\right)_{4}$ и $\mathrm{TbFe}_{2.5} \mathrm{Ga}_{0.5}\left(\mathrm{BO}_{3}\right)_{4}$ мягкие моды и структурные фазовые переходы были изучены ранее [9,32]. Чтобы получить более полную картину зависимости температуры фазовых переходов при замещении ионов железа на ионы галлия, мы повторили исследования этих двух кристаллов и включили в исследование два новых кристалла: $\mathrm{TbFe}_{2.45} \mathrm{Ga}_{0.55}\left(\mathrm{BO}_{3}\right)_{4}$ и $\mathrm{TbFe}_{2.4} \mathrm{Ga}_{0.6}\left(\mathrm{BO}_{3}\right)_{4}$.

Поведение спектров с изменением температуры в области низких частот представлено на рис. 1. В кристалле $\mathrm{TbFe}_{3}\left(\mathrm{BO}_{3}\right)_{4}$ ниже точки структурного фазового перехода наблюдается восстановление одной мягкой моды (рис. 1,a) (на рисунке мягкие моды отмечены звездочками). На карте интенсивности (рис. 2) кристалла $\mathrm{TbFe}_{3}\left(\mathrm{BO}_{3}\right)_{4}$ хорошо видно, что интенсивность этой линии вблизи перехода уменьшается почти до нуля, при этом ее частота нуля не достигает. Это объясняется тем, что данная мода появляется вследствие сворачивания зоны Бриллюэна при понижении симметрии от $R 32$ до $P 3_{1} 21$ с утроением объема элементарной ячейки и 

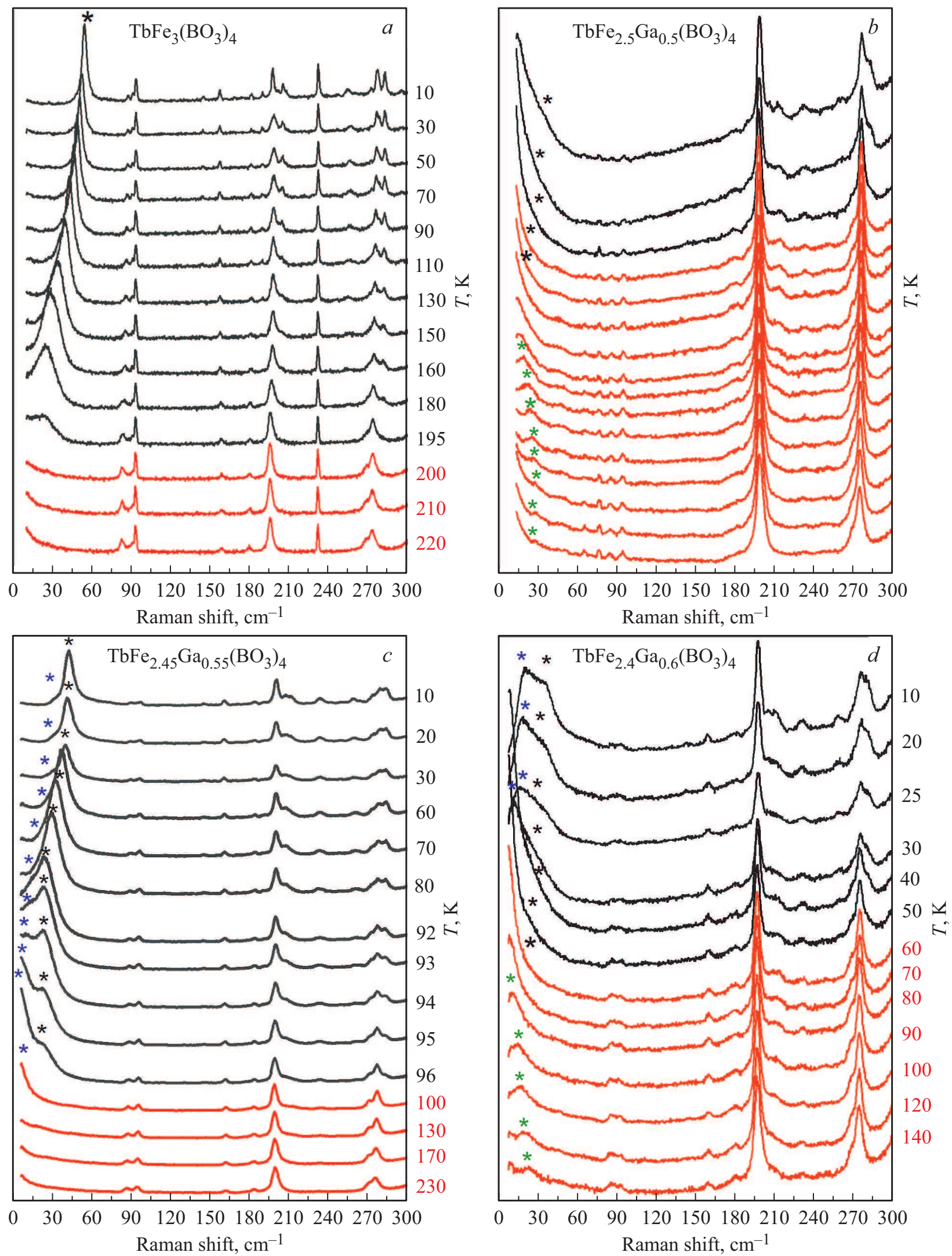

Рис. 1. Температурная трансформация спектров КРС в низкочастотном диапазоне для твердых растворов (по закладке): (a) $\mathrm{TbFe}_{3}\left(\mathrm{BO}_{3}\right)_{4},(b) \mathrm{TbFe}_{2.5} \mathrm{Ga}_{0.5}\left(\mathrm{BO}_{3}\right)_{4},(c) \mathrm{TbFe}_{2.45} \mathrm{Ga}_{0.55}\left(\mathrm{BO}_{3}\right)_{4},(d) \mathrm{TbFe}_{2.55} \mathrm{Ga}_{0.45}\left(\mathrm{BO}_{3}\right)_{4}$. Звездочками обозначены мягкие моды. Высокотемпературная фаза обозначена красным цветом, низкотемпературная - черным. 


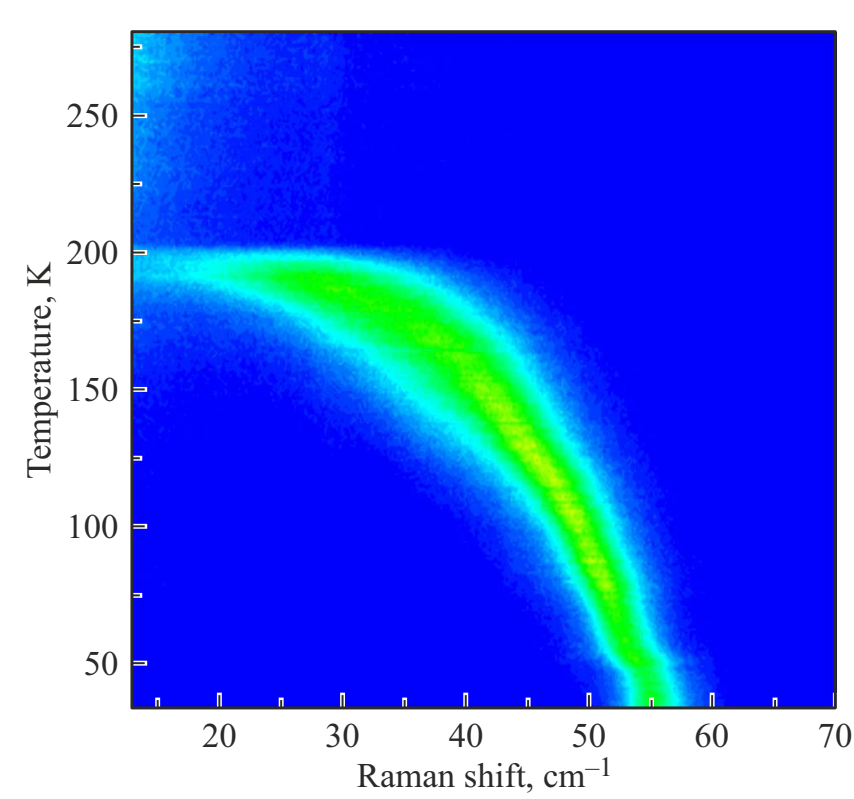

Рис. 2. Карта интенсивности в низких частотах в кристалле $\mathrm{TbFe}_{3}\left(\mathrm{BO}_{3}\right)_{4}$.

связанного с этим перехода фонона с акустической ветви на край зоны [9]. Ниже $50 \mathrm{~K}$ наблюдается смещение частоты мягкой моды, связанное с магнитоупругим взаимодействием между структурным и магнитным параметрами порядка. Подобное поведение мягкой моды ранее наблюдалось в кристалле $\mathrm{HoFe}_{2.5} \mathrm{Ga}_{0.5}\left(\mathrm{BO}_{3}\right)_{4}$ [40]. Температура структурного фазового перехода, определенная из поведения спектральных линий в этом кристалле, равна $198 \mathrm{~K}$, что согласуется с результатами, опубликованными ранее [9].

В спектрах кристалла $\mathrm{TbFe}_{2.5} \mathrm{Ga}_{0.5}\left(\mathrm{BO}_{3}\right)_{4}$ (в отличие от предыдущего кристалла) наблюдается как конденсация, так и восстановление мягкой моды (рис. 1,b). Мягкая мода конденсируется при охлаждении в высокосимметричной фазе и затем восстанавливается при дальнейшем охлаждении ниже фазового перехода от 33 до $10 \mathrm{~K}$.

Обычно в силу симметрийных ограничений в спектрах КРС наблюдается только процесс восстановления мягкой моды в низкосимметричной фазе после перехода. В данном случае такое поведение можно объяснить как результат нарушения симметрии кристалла, вызванного сильным взаимодействием структурного параметра порядка с магнитной подрешеткой из-за близости температур структурного и магнитного переходов в смешанных составах. Такие близкие температуры этих переходов в других боратах со структурой хантита ранее не наблюдались. Характерно, что для чистого состава конденсация мягкой моды в спектре КРС высокосимметричной фазы не наблюдается, что согласуется с результатами теоретико-группового анализа и численного моделирования.

Температура структурного перехода в кристалле $\mathrm{TbFe}_{2.5} \mathrm{Ga}_{0.5}\left(\mathrm{BO}_{3}\right)_{4}$ равна $33 \mathrm{~K}$ [32]. На карте интенсив- ности кристалла $\mathrm{TbFe}_{2.5} \mathrm{Ga}_{0.5}\left(\mathrm{BO}_{3}\right)_{4}$ кроме конденсации мягкой моды мы наблюдаем также восстановление второй мягкой моды при повышении температуры (рис. 3).

В кристалле твердого раствора $\mathrm{TbFe}_{2.45} \mathrm{Ga}_{0.55}\left(\mathrm{BO}_{3}\right)_{4}$ мы видим восстановление двух близких низкочастотных мод (рис. 1,c). При этом частота одной моды при приближении к температуре фазового перехода $T_{c}$ стремится к нулю, а движение второй моды останавливается за несколько градусов до перехода, затем ее интенсивность уменьшается, и при температуре $T_{c}$ она полностью исчезает. В этом кристалле температура

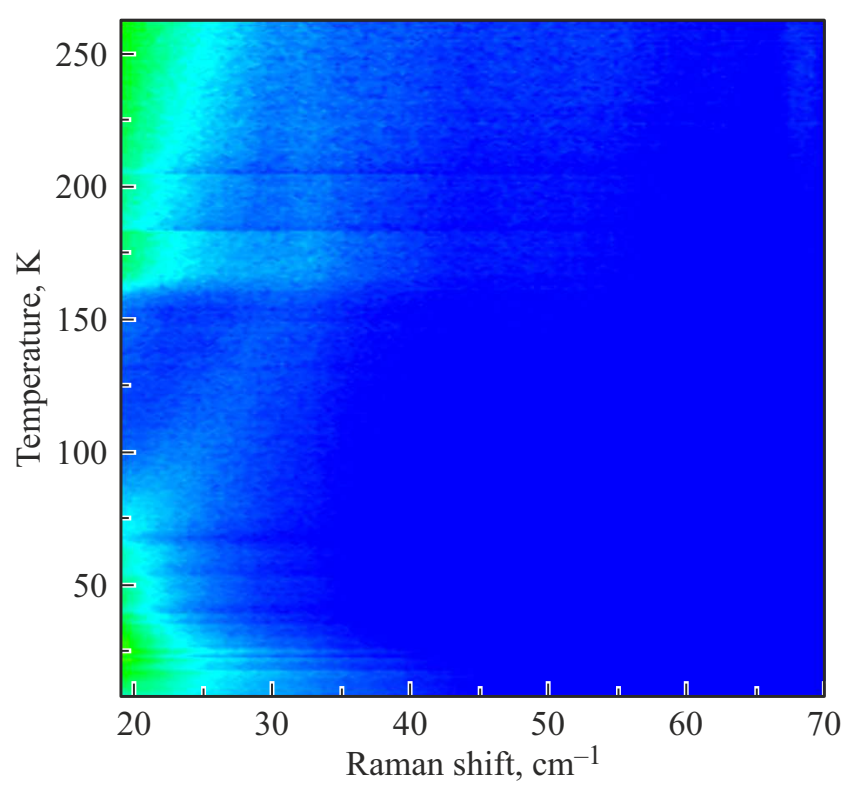

Рис. 3. Карта интенсивности в низких частотах в кристалле $\mathrm{TbFe}_{2.5} \mathrm{Ga}_{0.5}\left(\mathrm{BO}_{3}\right)_{4}$ (по закладке).

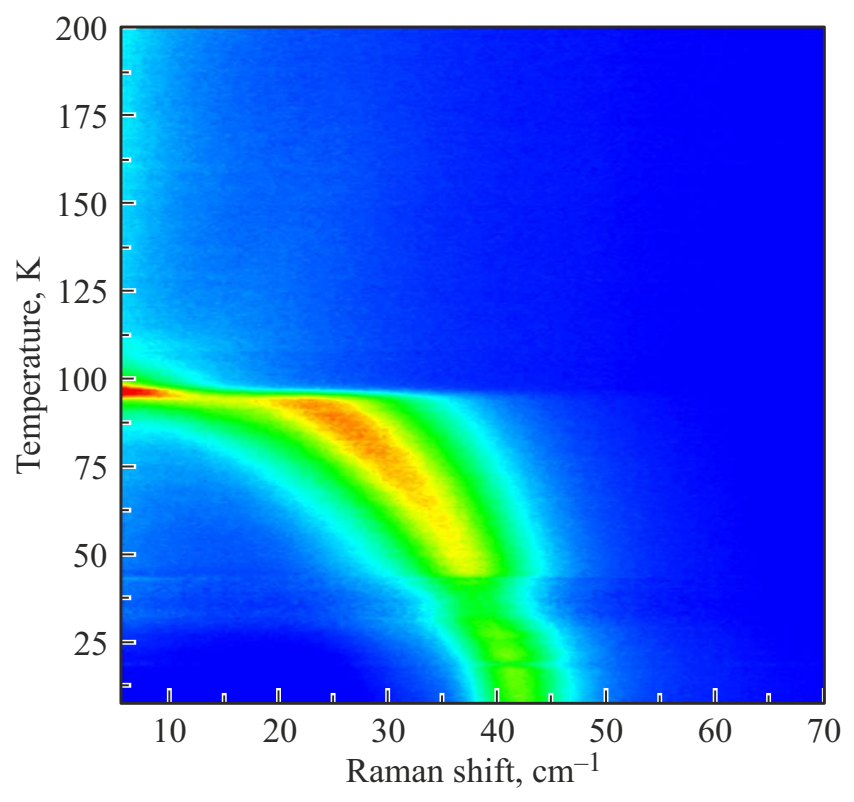

Pис. 4. Карта интенсивности в низких частотах в кристалле $\mathrm{TbFe}_{2.45} \mathrm{Ga}_{0.55}\left(\mathrm{BO}_{3}\right)_{4}$ (по закладке). 


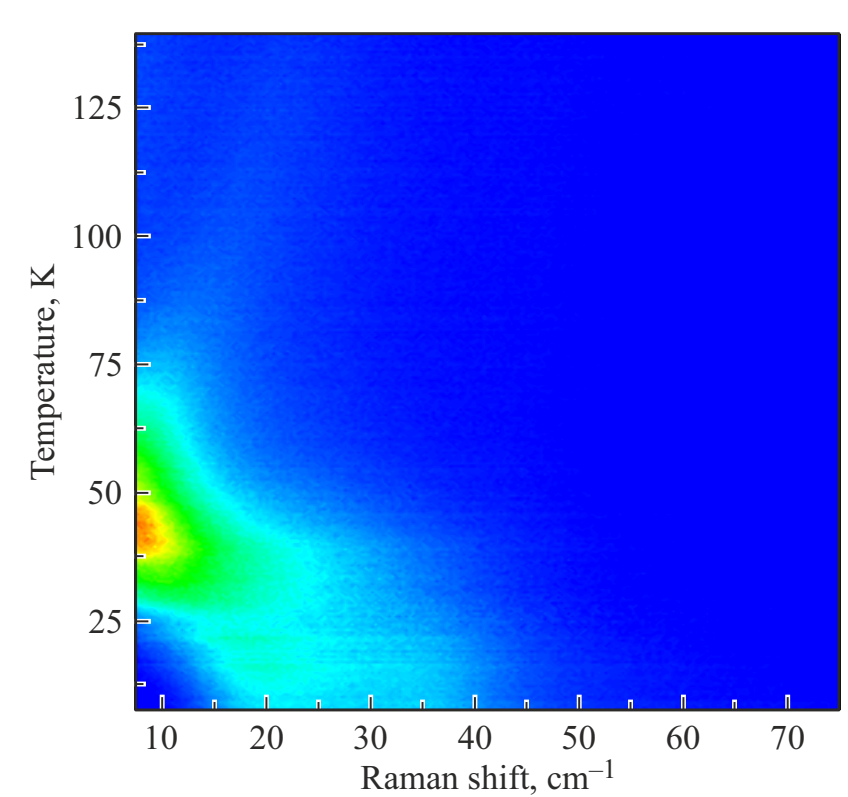

Рис. 5. Карта интенсивности в низких частотах в кристалле $\mathrm{TbFe}_{2.4} \mathrm{Ga}_{0.6}\left(\mathrm{BO}_{3}\right)_{4}$ (по закладке). Температура структурного перехода $(56 \mathrm{~K})$ предположительно близка к температуре магнитного перехода (еще не определена) твердого раствора.

структурного перехода $T_{c}=97 \mathrm{~K}$. На температурной карте интенсивностей спектральных линий кристалла $\mathrm{TbFe}_{2.45} \mathrm{Ga}_{0.55}\left(\mathrm{BO}_{3}\right)_{4}$ (рис. 4) конденсация двух близко расположенных мод видна по увеличению спектральной области, занимаемой линиями по мере приближения к температуре перехода. Остановка движения одной линии приводит к возникновению перетяжки на карте интенсивностей в области частот около $15 \mathrm{~cm}^{-1}$. Также можно заметить влияние магнитоупругого взаимодействия на поведение мягкой моды ниже $40 \mathrm{~K}$ (рис. 4).

В спектрах кристалла $\mathrm{TbFe}_{2.4} \mathrm{Ga}_{0.6}\left(\mathrm{BO}_{3}\right)_{4}$ мы также наблюдаем конденсацию одной низкочастотной моды при понижении температуры к точке фазового перехода и последующее восстановление двух близких мод (рис. $1, d$ ) при дальнейшем охлаждении. Температура фазового перехода $T_{c}=56 \mathrm{~K}$. На карте интенсивности кристалла $\mathrm{TbFe}_{2.4} \mathrm{Ga}_{0.6}\left(\mathrm{BO}_{3}\right)_{4}$ (рис. 5) можно увидеть малоинтенсивный пик, конденсирующийся к переходу, и две близкие восстанавливающиеся моды при дальнейшем охлаждении кристалла. Рентгеноструктурные исследования, выполненные на образцах из той же кристаллизации, однозначно указывают на переход из фазы $R 32$ в фазу $P 3_{1} 21$ при понижении температуры. В целом поведение мягких мод в этом твердом растворе похоже на наблюдавшееся в твердом растворе $\mathrm{TbFe}_{2.45} \mathrm{Ga}_{0.55}\left(\mathrm{BO}_{3}\right)_{4}$.

Структурный фазовый переход из фазы $R 32$ в фазу $P 3_{1} 21$ в кристалле $\mathrm{TbFe}_{3}\left(\mathrm{BO}_{3}\right)_{4}$, согласно расчету, связан с конденсацией фононной моды в точке зоны Бриллюэна с последующим „сворачиванием“ зоны. В результате этого фононы из точки $\Lambda$ высокотемпературной фазы оказываются в точке $\Gamma$ зоны Бриллюэна низкотемпературной фазы [15]. Экспериментально в спектрах КРС кристаллов смешанных составов мы наблюдаем две низкочастотных моды: до и после перехода. Такое поведение возможно, если мы наблюдаем мягкую моду из точки $\Lambda$ зоны Бриллюэна высокотемпературной фазы вследствие нарушения правил отбора, которое, вероятно, вызвано сильным взаимодействием с магнитной подрешеткой из-за близости температур структурного и магнитного переходов и неупорядоченностью решеток смешанных кристаллов.

При рассмотрении отдельного спектра при одной конкретной температуре на спектре КРС практически невозможно заметить линию, соответствующую „мягкой“ моде в фазе $R 32$, однако ее температурное поведение и движение очень хорошо видны на последовательной анимации температурных спектров и температурных картах интенсивности рассеяния. Очень слабая интенсивность этой моды не позволяет выполнить разложение контура с последующем представлением зависимости положения линии или ее параметров от температуры.

Анализ зависимости температур структурных фазовых переходов от состава показал отсутствие монотонной зависимости между закладкой галлия при синтезе твердых растворов и температурой фазовых переходов. Поведение частот мягких мод также не коррелирует с концентрацией галлия в закладках. Для прояснения вопроса существования зависимости температур переходов от содержания галлия в кристаллах было выполнено уточнение составов твердых растворов.

Дополнительно были проведены исследования методом рентгеновской энергодисперсионной микроскопии, и по результатам определено содержание галлия во всех исследуемых в работе кристаллов. Результаты уточнения концентрации ионов железа и галлия состава твердых растворов представлены в табл. 2.

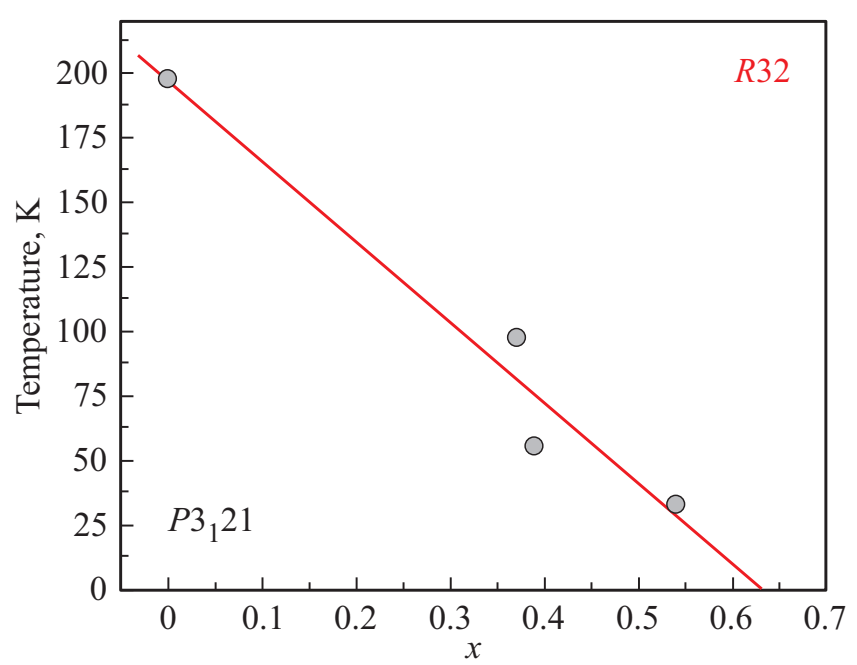

Рис. 6. Фазовая диаграмма: зависимость температуры структурного перехода в твердых растворах $\mathrm{TbFe}_{3-x} \mathrm{Ga}_{x}\left(\mathrm{BO}_{3}\right)_{4}$ от содержания галлия. 
Таблица 2. Уточнение содержания галлия в твердых раствоpax $\mathrm{TbFe}_{3-x} \mathrm{Ga}_{x}\left(\mathrm{BO}_{3}\right)_{4}$ и температуры структурного перехода

\begin{tabular}{c|c|c}
\hline $\begin{array}{c}\text { Формула } \\
\text { по закладке при росте }\end{array}$ & $\begin{array}{c}\text { Формула } \\
\text { по результату РЭМ }\end{array}$ & $T, \mathrm{~K}$ \\
\hline $\mathrm{TbFe}_{3}\left(\mathrm{BO}_{3}\right)_{4}$ & $\mathrm{TbFe}_{3}\left(\mathrm{BO}_{3}\right)_{4}$ & 198 \\
\hline $\mathrm{TbFe}_{2.5} \mathrm{Ga}_{0.5}\left(\mathrm{BO}_{3}\right)_{4}$ & $\mathrm{TbFe}_{2.46} \mathrm{Ga}_{0.54}\left(\mathrm{BO}_{3}\right)_{4}$ & 33 \\
\hline $\mathrm{TbFe}_{2.45} \mathrm{Ga}_{0.55}\left(\mathrm{BO}_{3}\right)_{4}$ & $\mathrm{TbFe}_{2.63} \mathrm{Ga}_{0.37}\left(\mathrm{BO}_{3}\right)_{4}$ & 97 \\
\hline $\mathrm{TbFe}_{2.4} \mathrm{Ga}_{0.6}\left(\mathrm{BO}_{3}\right)_{4}$ & $\mathrm{TbFe}_{2.61} \mathrm{Ga}_{0.39}\left(\mathrm{BO}_{3}\right)_{4}$ & 56
\end{tabular}

По уточненным данным и определенным ранее температурам фазовых переходов построена фазовая диаграмма „Состав-Температура“ (рис. 6). Ошибка определения границы раздела фаз (на основе полученных данных) составила 9\%. Кроме того, РЭМ-анализ показал, что в образцах $\mathrm{TbFe}_{2.46} \mathrm{Ga}_{0.54}\left(\mathrm{BO}_{3}\right)_{4}$ и $\mathrm{TbFe}_{2.4} \mathrm{Ga}_{0.6}\left(\mathrm{BO}_{3}\right)_{4}$ в составе обнаруживалось небольшое вхождение атомов $\mathrm{Bi}$, тогда как в позднее синтезированных кристаллах с составами $\mathrm{TbFe}_{2.46} \mathrm{Ga}_{0.54}\left(\mathrm{BO}_{3}\right)_{4}$ и $\mathrm{TbFe}_{2.4} \mathrm{Ga}_{0.6}\left(\mathrm{BO}_{3}\right)_{4}$ вхождения атомов Ві в состав обнаружено не было.

Изменения состава твердых кристаллов незначительно влияет на изменение температуры магнитного упорядочения структуры, которая для исследованных составов лежит в диапазоне $35-50 \mathrm{~K}$. Приблизительно температуры магнитного упорядочения можно также определить по аномалиям в спектрах КРС в высокочастотном диапазоне. Согласно этим данным, при концентрации атомов $\mathrm{Ga}$ в составе на уровне $x=0.5-0.6$ возможно получить кристаллы твердых растворов, в которых температуры структурного и магнитного переходов будут очень близки или совпадут. Это может привести к очень сильному взаимодействию структурного и магнитного параметров порядка (близких по температуре мягких мод). Такие кристаллы могут стать хорошими модельными объектами для изучения магнитоупругих взаимодействий в мультиферроиках.

\section{Заключение}

В настоящей работе были синтезированы монокристаллы твердых растворов $\mathrm{TbFe}_{3-x} \mathrm{Ga}_{x}\left(\mathrm{BO}_{3}\right)_{4} \quad(x=0$, $0.54,0.39,0.37)$. Концентрация ионов $\mathrm{Fe}$ и $\mathrm{Ga}$ в синтезированных кристаллах твердых растворов уточнена методом РЭМ. Выполнены температурные исследования монокристаллов твердых растворов методом спектроскопии КРС. Во всех исследуемых образцах $\mathrm{TbFe}_{3}\left(\mathrm{BO}_{3}\right)_{4}, \mathrm{TbFe}_{2.46} \mathrm{Ga}_{0.54}\left(\mathrm{BO}_{3}\right)_{4}, \mathrm{TbFe}_{2.61} \mathrm{Ga}_{0.39}\left(\mathrm{BO}_{3}\right)_{4}$, $\mathrm{TbFe}_{2.63} \mathrm{Ga}_{0.37}\left(\mathrm{BO}_{3}\right)_{4}$ наблюдались спектральные аномалии, характерные для восстановления мягких мод в низкосимметричной фазе. Обнаружено, что при наличии и с увеличением замещения ионов $\mathrm{Fe}$ на ионы $\mathrm{Ga}$ в кристаллах $\mathrm{TbFe}_{2.63} \mathrm{Ga}_{0.37}\left(\mathrm{BO}_{3}\right)_{4}, \mathrm{TbFe}_{2.61} \mathrm{Ga}_{0.39}\left(\mathrm{BO}_{3}\right)_{4}$ и $\mathrm{TbFe}_{2.46} \mathrm{Ga}_{0.54}\left(\mathrm{BO}_{3}\right)_{4}$ в спектрах дополнительно ста- новится видна еще одна восстанавливающая низкочастотная мода, а также в высокосимметричной фазе $R 32$ активируется низкочастотная мода, конденсирующаяся при охлаждении кристалла к точке структурного фазового перехода. Наблюдалось значительное влияние замещения ионов железа на ионы галлия в твердых растворах $\mathrm{TbFe}_{3-x} \mathrm{Ga}_{x}\left(\mathrm{BO}_{3}\right)_{4}$ на температуру структурного фазового перехода. Увеличение концентрации ионов $\mathrm{Ga}$ в твердых растворах приводит к понижению температуры структурного перехода из фазы $R 32$ в фазу $P 3_{1} 21$; в кристалле $\mathrm{TbFe}_{2.46} \mathrm{Ga}_{0.54}\left(\mathrm{BO}_{3}\right)_{4}$ температуры структурного перехода и перехода магнитного упорядочения практически совпадают. В температурных зависимостях востанавливающихся низкочастотных мод наблюдаются небольшие аномалии, связанные с взаимодействием магнитоупругого и структурного параметров порядка.

\section{Благодарности}

При проведении исследований использовалось оборудование Красноярского регионального центра коллективного пользования ФИЦ КНЦ СО РАН

\section{Финансирование работы}

Авторы благодарны РФФИ, Правительству Красноярского края и Красноярского краевого фонда науки за финансирование в рамках научного проекта грант № 20-42-240009 p_a_Красноярск, а также РФФИ (Российский фонд фундаментальных исследований) и Немецкого научно-исследовательского сообщества (Deutsche Forschungsgemeinschaft) за финансовую поддержку в рамках проекта № 21-52-12018 ННИО_а.

\section{Конфликт интересов}

Авторы заявляют об отсутствии конфликта интересов.

\section{Список литературы}

[1] G.M. Kuz'micheva, I.A. Kaurova, V.B. Rybakov, $\begin{array}{lllll}\text { V.V. Podbel'skiy. Crystals, 9, } 100 & \text { (2019). }\end{array}$ DOI: $10.3390 /$ cryst9020100

[2] M. Fiebig. J. Phys. D.: Appl. Phys., 38, R123 (2005). DOI: $10.1088 / 0022-3727 / 38 / 8 / \mathrm{R} 01$

[3] A.M. Kadomtseva, Yu.F. Popov, G.P. Vorob'ev, A.P. Pyatakov, S.S. Krotov, K.I. Kamilov, V.Yu. Ivanov, A.A. Mukhin, A.K. Zvezdin, A.M. Kuz'menko, L.N. Bezmaternykh, I.A. Gudim, V.L. Temerov. Low Temperature Physics, 36, 511 (2010). DOI: 10.1063/1.3457390

[4] A.N. Vasiliev, E.A. Popova. Low Temperature Physics, 32, 735 (2006). DOI: 10.1063/1.2219496

[5] W. Eerenstein, N.D. Mathur, J.F. Scott. Nature, 442, 759 (2006). DOI: $10.1038 /$ nature 05023

[6] M.M. Vopson. Critical Reviews in Solid State and Materials Sciences, $\quad \mathbf{4 0}(4), \quad 223 \quad$ (2015). DOI: $10.1080 / 10408436.2014 .992584$ 
[7] N.I. Leonyuk, L.I. Leonyuk. Prog. Crystal Growth. and Charact., 31, 179 (1995).

[8] L. Bezmaternykh, V. Temerov, I. Gudim, N. Stolbovaya. Crystallography Reports, 50, s97 (2005). DOI: $10.1134 / 1.2133981$

[9] D. Fausti, A.A. Nugroho, P.H.M. van Loosdrecht, S.A. Klimin, M.N. Popova, L.N. Bezmaternykh. Phys. Rev. B, 74, 024403 (2006). DOI: 10.1103/PhysRevB.74.024403

[10] C. Ritter, A. Balaev, A. Vorotynov, G. Petrakovskii, D. Velikanov, V. Temerov, I. Gudim. J. Phys.: Condens. Matters, 19, 196227 (2007). DOI: 10.1088/0953$8984 / 19 / 19 / 196227$

[11] V.V. Kurnosov, V.S. Tsapenko, L.N. Bezmaternykh, I.A. Gudim. Low Temperature Physics, 40 (12), 1087 (2014). DOI: $10.1063 / 1.4904002$

[12] Y. Hinatsu, Y. Doi, K. Ito, M. Wakeshima, A. Alemi. J. Solid State Chem., 172, 438 (2003). DOI: 10.1016/S00224596(03)00028-8

[13] E. Moshkina, S. Krylova, I. Gudim, M. Molokeev, V. Temerov, M. Pavlovskiy, A. Vtyurin, A. Krylov. Cryst. Growth Des., 20, 1058 (2020). DOI: 10.1021/acs.cgd.9b01387

[14] E.A. Popova, D.V. Volkov, A.N. Vasiliev, A.A. Demidov, N.P. Kolmakova, I.A. Gudim, L.N. Bezmatenykh, N. Tristan, Yu. Skourski, B. Büchner, C. Hess, R. Klingeler. Phys. Rev. B, 75, 224413 (2007). DOI: 10.1103/PhysRevB.75.224413

[15] M.S. Pavlovskiy, K.A. Shaykhutdinov, L.S. Wu, G. Ehlers, V.L. Temerov, I.A. Gudim, A.S. Shinkorenko, A. Podlesnyak. Phys. Rev. B, 97, 054313 (2018) DOI: 10.1103/PhysRevB.97.054313

[16] D.V. Volkov, E.A. Popova, N.P. Kolmakov, A.A. Demidov, N. Tristan, Yu. Skourski, B. Buechner, I.A. Gudim, L.N. Bezmaternykh. J. Magn. Magn. Mater., 316, e717 (2007). DOI: $10.1016 /$ j.jmmm.2007.03.070

[17] T.N. Stanislavchuk, E.P. Chukalina, L.N. Bezmaternykh. J. Opt. Technol., 74, 130 (2007). DOI: 10.1364/JOT.74.000139

[18] U. Adem, L. Wang, D. Fausti, W. Schottenhamel, P.H.M. van Loosdrecht, A. Vasiliev, L.N. Bezmaternykh, B. Büchner, C. Hess, R. Klingeler. Phys. Rev. B, 82, 064406 (2010). DOI: 10.1103/PhysRevB.82.064406

[19] A.V. Malakhovskii, S.L. Gnatchenko, I.S. Kachur, V.G. Piryatinskaya, A.L. Sukhachev, V.L. Temerov. Eur. Phys. J. B, 80, 1 (2011). DOI: 10.1140/epjb/e2011-10806-x

[20] M.N. Popova, T.N. Stanislavchuk, B.Z. Malkin, L.N. Bezmaternykh. J. Phys.: Condens. Matter, 24 (19), 196002 (2012). DOI: 10.1088/0953-8984/24/19/196002

[21] S.L. Gnatchenko, I.S. Kachur, V.G. Piryatinskaya, V.A. Bedarev, M.I. Pashchenko. Low Temp. Phys., 37, 693 (2011). DOI: 10.1063/1.3660219

[22] D. Szaller, V. Kocsis, S. Bordács, T. Fehér, T. Rõõm, U. Nagel, H. Engelkamp, K. Ohgushi, I. Kézsmárki. Phys. Rev. B, 95, 024427 (2017). DOI: 10.1103/PhysRevB.95.024427

[23] S. Krylova, I. Gudim, A. Aleksandrovsky, A. Vtyurin, A. Krylov. Ferroelectrics, $\mathbf{5 7 5}(1), \quad 11 \quad$ (2021). DOI: 10.1080/00150193.2021.1888219

[24] S.N. Krylova, A.S. Aleksandrovsky, E.M. Roginskii, A.A. Krylov, I.A. Gudim, A.N. Vtyurin. Ferroelectrics, 559 (1), 135 (2020). DOI: 10.1080/00150193.2020.1722015

[25] R.Z. Levitin, E.A. Popova, R.M. Chtsherbov, A.N. Vasiliev, M.N. Popova, E.P. Chukalina, S.A. Klimin, P.H.M. van Loosdrecht, D. Fausti, L.N. Bezmaternykh. JETP Lett., 79, 423 (2004). DOI: 10.1134/1.1776236
[26] S.A. Klimin, D. Fausti, A. Meetsma, L.N. Bezmaternykh, P.H.M. van Loosdrechta, T.T. Palstra. Acta Cryst. B, 61, 481 (2005). DOI: $10.1107 / \mathrm{S} 0108768105017362$

[27] A.S. Krylov, I.A. Gudim, I. Nemtsev, S.N. Krylova, A.V. Shabanov, A.A. Krylov. J. Raman Spectrosc., 48, 1406 (2017). DOI: $10.1002 /$ jrs.5078

[28] E. Moshkina, A. Krylov, S. Sofronova, I. Gudim, V. Temerov. Cryst. Growth Des., 16, 6915 (2016). DOI: $10.1021 /$ acs.cgd.6b01079

[29] E. Moshkina, I. Gudim, V. Temerov, A. Krylov. J. Raman Spectrosc., 49, 1732 (2018). DOI: 10.1002/jrs.5430

[30] A.S. Krylov, S.N. Sofronova, I.A. Gudim, A.N. Vtyurin. Solid State Commun., 174, 26 (2013). DOI: $10.1016 / j . s s c .2013 .09 .011$

[31] A.S. Krylov, S.N. Sofronova, I.A. Gudim, S.N. Krylova, R. Kumar, A.N. Vtyurin. J. Adv. Dielectr., 8, 1850011 (2018). DOI: 10.1142/S2010135X1850011X

[32] A.S. Krylov, I.A. Gudim, S.N. Krylova, A.A. Krylov, A.N. Vtyurin. Ferroelectrics, 559, 128 (2020). DOI: $10.1080 / 00150193.2020 .1722014$

[33] A.V. Peschanskii, A.V. Yeremenko, V.I. Fomin, L.N. Bezmaternykh, I.A. Gudim. Low Temperature Physics, 40, 171 (2014). DOI: 10.1063/1.4865566 17

[34] S.A. Klimin, A.B. Kuzmenko, M.A. Kashchenko, M.N. Popova. Phys. Rev. B, 93, 054304 (2016). DOI: 10.1103/PhysRevB.93.054304

[35] M.N. Popova. J. Magn. Magn. Mat., 321 (7), 716 (2009). DOI: 10.1016/j.jmmm.2008.11.033.

[36] Л.Н. Безматерных, С.А. Харламова, В.Л. Темеров. Кристаллография, 49 (4), 1 (2004).

[37] V.L. Ginzburg. Ferroelectrics, $\quad \mathbf{7 6}(1), \quad 3 \quad$ (1987) DOI: $10.1080 / 00150198708009019$

[38] L.D. Landau, E.M. Lifshitz. Statistical physics. Part 1 (Pergamon Press, Oxford, 1980).

[39] J.F. Scott. Rev. Mod. Phys. 46 (1), 83 (1974). DOI: 10.1103/RevModPhys.46.83

[40] A. Krylov, S. Krylova, I. Gudim, A. Vtyurin. Ferroelectrics, 556, 16 (2020). DOI: 10.1080/00150193.2020.1713334 